\section{Amniotic membrane transplantation in surgical management of ocular surface squamous neoplasias: long-term results}

\author{
Abstract \\ Objective To evaluate the long-term efficacy \\ of amniotic membrane transplantation for \\ ocular surface reconstruction in the surgical \\ management of ocular surface squamous \\ neoplasia (OSSN). \\ Methods OSSN in 21 patients (7 female, \\ 14 male patients) was managed with \\ excisional biopsy, cryotherapy, corneal \\ epitheliectomy with absolute alcohol \\ application when the cornea is involved, \\ lamellar sclerectomy and adjunctional \\ absolute alcohol application to the base \\ when episclera is involved, and ocular \\ surface reconstruction with cryopreserved \\ amniotic membrane transplantation. \\ Tumor control and complications were \\ evaluated. \\ Results The mean age of the patients \\ was $62.42 \pm 20.9$ (range, 16-84). The \\ average diameter of the base of the tumors \\ was $13.1 \pm 4.8$ (range, 9-21) $\mathrm{mm}$ and \\ complete removal was achieved in all cases \\ as revealed histopathologically. Ocular \\ surface healing was achieved in all cases. \\ At the postoperative period, limbal stem \\ cell deficiency in three eyes and mild \\ symblepharon in one eye were detected. \\ In a mean follow-up of $30.95 \pm 18.8$ (range, \\ 13-75) months, no recurrence was detected. \\ Conclusion For large or multifocal \\ conjunctival tumors, the reconstruction of \\ ocular surface and fornix is challenging. \\ The amniotic-membrane use to repair \\ conjunctival defects larger than $\mathbf{1 0} \mathbf{m m}$ is a \\ safe and effective technique with minimal \\ complications allowing surgeons to make \\ large enough excisions. \\ Eye (2014) 28, 1131-1135; doi:10.1038/eye.2014.148; \\ published online 4 July 2014
}

M Palamar ${ }^{1}$, E Kaya ${ }^{1}$, S Egrilmez ${ }^{1}$, T Akalin²

and A Yagci $^{1}$

\section{Introduction}

Ocular surface squamous neoplasia (OSSN) encompasses a broad spectrum of neoplastic squamous epithelial abnormalities, including squamous dysplasia, conjunctival intraepithelial neoplasia (CIN), and invasive squamous cell carcinoma (SCC). ${ }^{1,2}$ These neoplastic conditions can affect the conjunctiva and the corneal surface, and occasionally invade into the globe, orbit, and the nasolacrimal system. ${ }^{1-3}$ In a survey on 1643 conjunctival tumors by Shields et al, 179 tumors (11\%) were classified under the category of OSSN. The proposed etiologic risk factors for OSSN include environmental exposure to UV-B solar radiation, cigarette smoke, human papilloma virus, human immunodeficiency virus, petroleum products, medical immunosuppressive agents for organ transplant, and corneal graft. ${ }^{4,5}$

Complete tumor resection to avoid destructive local invasion, recurrence, and metastasis is an essential issue in the treatment of OSSN. However, reconstruction of large excised areas ensuring both good functional and cosmetic results is as much important and can be very challenging. When the wound cannot be closed primarily, several surgical techniques might be used for the management of these tissue defects. All of these methods, including amniotic membrane transplantation (AMT), transpositional or free flap conjunctival autografting, limbal transplantation, and oral mucosa transplantation have their advantages as well as limitations. ${ }^{6}$

The use of fetal membrane allografts for ophthalmic purposes was first reported by de Rotth in $1940 .^{7}$ AMT was introduced by Tseng and colleagues ${ }^{8,9}$ for the management
${ }^{1}$ Department of Ophthalmology, Ege University Faculty of Medicine, Izmir, Turkey

${ }^{2}$ Department of Pathology, Ege University Faculty of Medicine, Izmir, Turkey

Correspondence: M Palamar, Ege Universitesi Tip Fakultesi Goz Hastaliklari, Bornova, Izmir AD 35040, Turkey Tel/Fax: + 90232 3881469; E-mail: melispalamar@ hotmail.com

Received: 20 December 2013

Accepted in revised form: 20 May 2014

Published online:

4 July 2014 
of patients with corneal defects, pterygia, neoplasia, and symblepharon. The amniotic membrane (AM) promotes epithelization by functioning as a substrate for growth of epithelial cells. It also serves as an antiangiogenic, anti-inflammatory, and antifibrotic agent with the help of its structural and biochemical composition. ${ }^{10}$ The techniques to harvest and preserve human AM have been well established. ${ }^{8-10}$ The use of this graft is safe, as long as the preparation is carried out according to a standard protocol. ${ }^{8-10}$

A number of studies have been conducted regarding AMT for ocular surface reconstruction in ocular surface neoplasias. ${ }^{6,9-12}$ However, long-term results of the technique is still not published. Herein, we present our long-term experience (over 13 months up to 75 months) and results of OSSN treatment using cryopreserved human AM for ocular surface reconstruction. The results of 21 cases of OSSN excision followed by AMT to reconstruct the ocular surface are reported.

\section{Materials and methods}

From February 2006 to September 2012, 21 patients (21 eyes) who underwent excision of OSSN followed by AMT were enroled in the study. Surgeries were performed in the same clinic by the same surgeons. During each follow-up visit, slit-lamp biomicroscopy and fluorescein staining were performed, noting the integrity, smoothness, and wetness of the ocular surface, any signs of any complications (inflammation, scarring,

vascularization, and corneal opacities), and recurrences. The records, photographs of the ocular surface, and histopathologic slides were retrospectively analyzed. Treatment results were defined as successful if complete epithelization of the surgical wound was achieved and if there were no clinically significant complications or recurrences of the tumor.

All operations were performed under local anesthesia. All tumors were resected intact, using the 'no touch' technique with at least 2-mm normal-looking conjunctiva (safe margins). ${ }^{13,14}$ All excised tissues were sent for histopathologic evaluation to the pathology department. Double freeze-thaw cryotherapy was applied to the remaining conjunctival margins. For tumors invading the cornea before tumor excision corneal epitheliectomy with absolute alcohol application was performed. For tumors invading the sclera lamellar, sclerectomy with adjunctive absolute alcohol application to the base of the excised tumor was performed. The conjunctival defects were reconstructed up to the limbus with one sheet of cryopreserved single layer AM allograft-the stromal side facing down-secured side to side with $8 / 0$ vicryl sutures. The largest diameter of the AM was ranging from 13 to $24 \mathrm{~mm}$ according to the defect size. While suturing, attention was paid to avoid tension on the AM graft. A pressure patch was applied for 3 days. Topical corticosteroid and antibiotic eye drops were used four times daily for a month. No adjunctional treatment, such as mitomycin C or interferon alfa-2b, were applied.

\section{Results}

The mean age of the 21 patients ( 7 female, 14 male patients) was $62.42 \pm 20.9$ (range, 16-84). The right eye was effected in 11, and the left eye was effected in 10 patients. The effected quadrants were nasal (nine eyes), temporal (nine eyes), superior (one eye), inferior (one eye), and temporal-superior-inferior (one eye). All tumors were primary and did not receive any prior treatment. None of the patients had any local invasions to the orbit or any local lymph node metastasis at the time of surgery.

Complete tumor removal confirmed using histopatology was achieved in all cases (100\%). The average diameter of the base of the tumors was $13.1 \pm 4.8$ (range, 9-21) mm. Bulbar conjunctiva was involved in all cases. The tumors invaded the limbal area in 18 cases $(85.7 \%)$. The mean extent of the limbal involvement was $4 \pm 2.5$ (range, 2-8) clock hours. In 14 cases (66.6\%) the tumor infiltrated the cornea and corneal epitheliectomy was also needed. In four cases $(19.0 \%)$ the sclera was found to be involved and lamellar sclerectomy was also performed. Pathologic diagnosis of the tumors were squamous dysplasia (two eyes), CIN (nine eyes), and SCC (ten eyes).

No surgical complications were observed. Postoperatively, the AM completely covered the surgical defect of the ocular surface in all cases. Transient signs of irritation in the AM graft area and mild discomfort lasted for 2-3 weeks. The mean follow-up time was $30.9 \pm 18.8$ (range, 13-75) months. At the last follow-up visit, the patients did not report any symptoms associated with the surgery and were satisfied with the cosmetic appearance of their eyes. In all cases, a final slit-lamp examination with fluorescein staining showed complete epithelization of the tissue defect; a noninflamed and wet surface of conjunctiva and cornea was observed (Figures 1a-f). Partial limbal stem cell deficiency that did not interfere the visual acuity was found in three eyes (14.3\%; Figures $2 \mathrm{a}-\mathrm{c})$. A superomedial symblepharon was diagnosed in one eye $(4.7 \%)$ after the excision of the tumor; however, it was not clinically significant (Figures 2d-f). All eyes except the one with symblepharon showed a smooth ocular surface. No recurrence was observed in a follow-up time of $30.95 \pm 18.8$ (range, 13-75) months. 


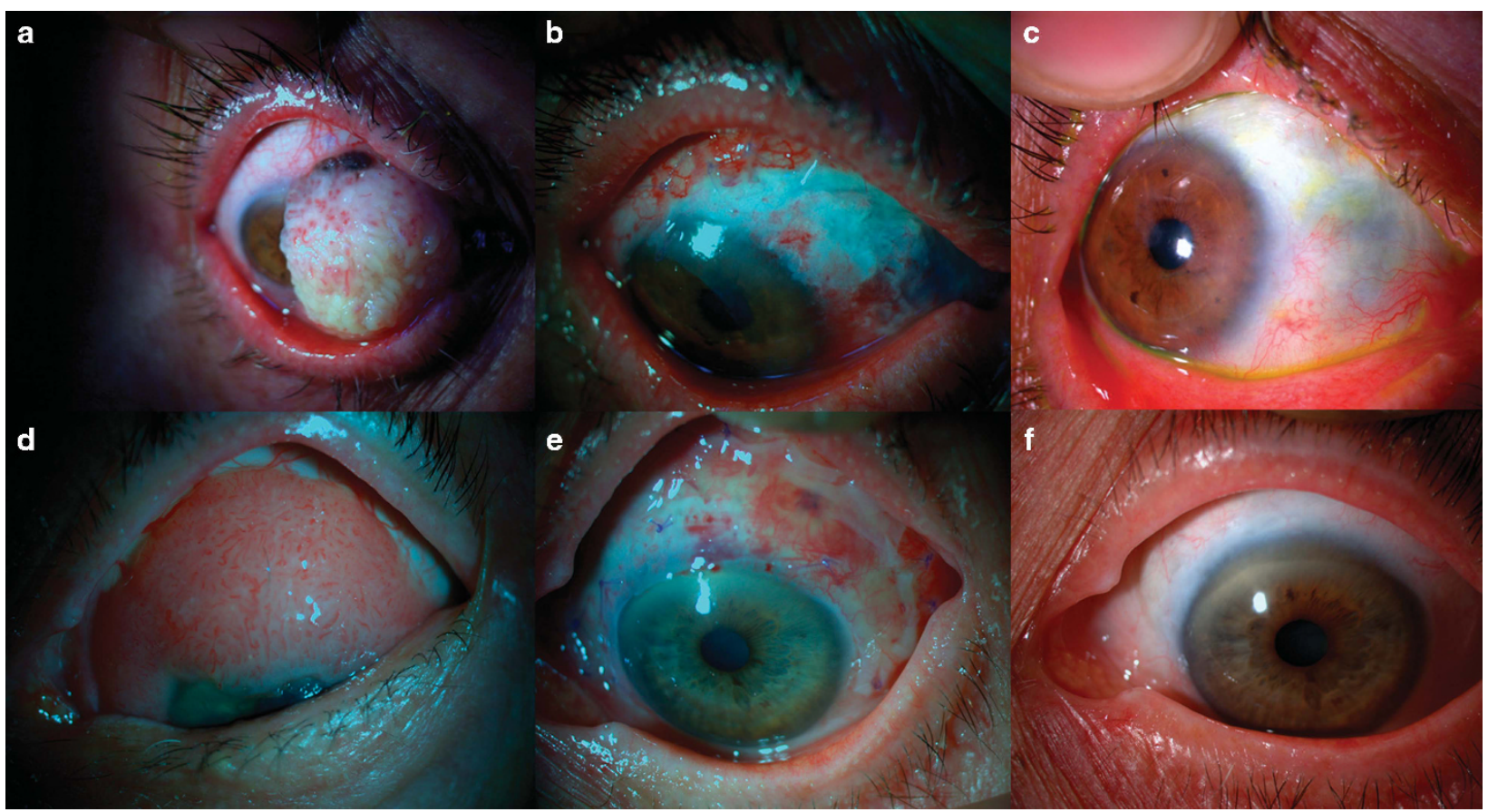

Figure 1 (a) Preoperative picture of a 70-year-old female patient with squamous neoplasia involving two-thirds of the cornea. (b) Early postoperative picture showing the amniotic membrane. (c) Late postoperative (30 months) appearance of the eye. (d) Preoperative picture of an 84-year-old female with conjunctival intraepithelial neoplasia involving the cornea. (e) Early postoperative picture showing the ocular surface and amniotic membrane. (f) Late postoperative (24 months) appearance of the eye.

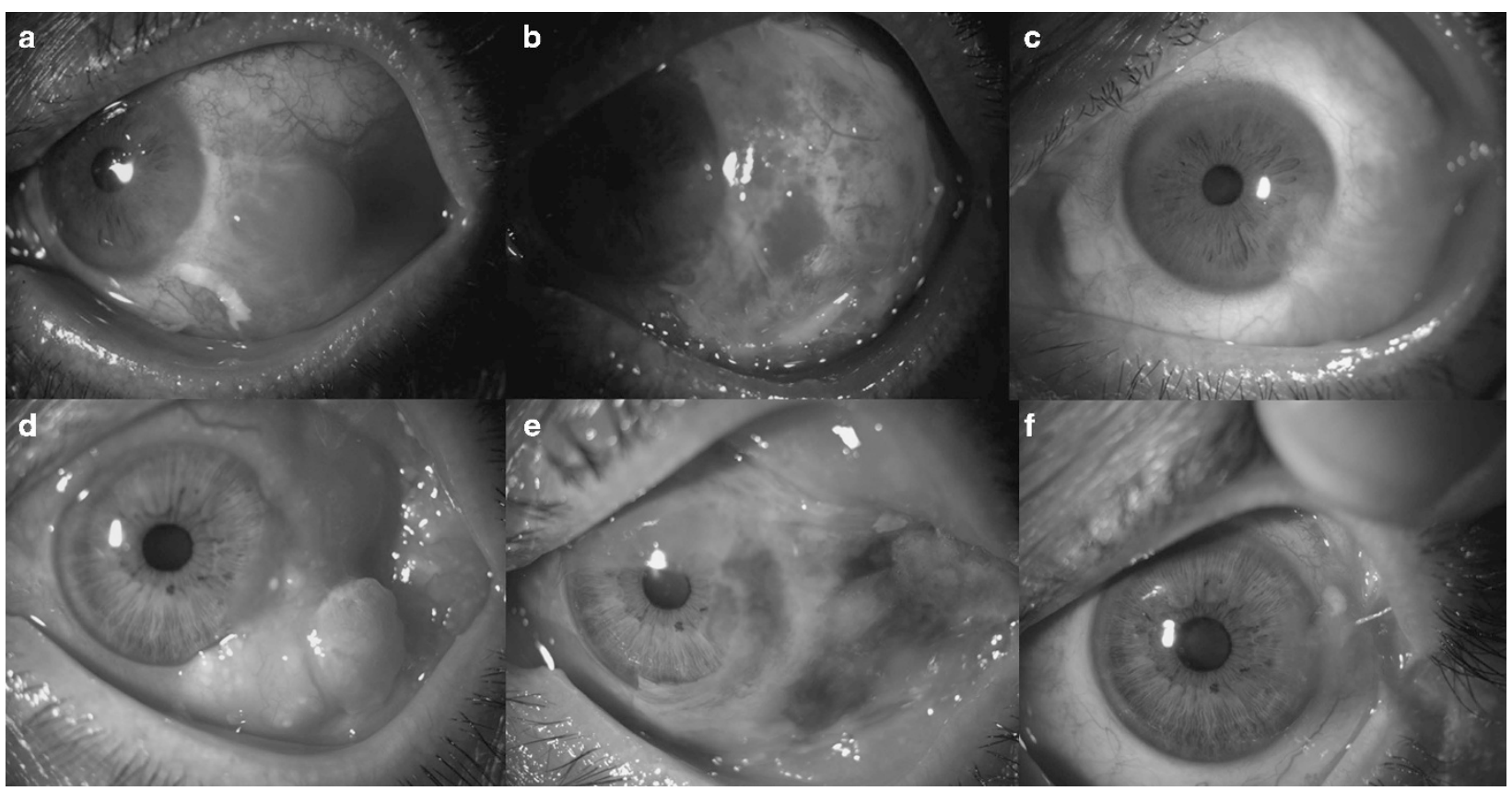

Figure 2 (a) Preoperative picture of a 55-year-old male patient with squamous neoplasia. (b) Early postoperative picture showing the ocular surface. (c) Late postoperative ( 25 months) appearance of the eye to demonstrate the inferotemporal limbal stem cell deficiency. (d) Preoperative picture of a 60-year-old male with squamous neoplasia. (e) Early postoperative picture showing the amniotic membrane. (f) Late postoperative (18 months) appearance of the eye demonstrating mild superonasal symblepharon. 


\section{Discussion}

Surgical excision of OSSN larger than 10-mm base diameter might result in wide tissue defects that cannot be closed primarily. These defects require reconstruction with adjunctive methods, such as transpositional conjunctival flap, free conjunctival graft from the opposite eye, and/or oral mucosal grafts. The main handicap of autologous grafts and flaps is the shortage of the utilizable tissue. Moreover, in case of conjunctival grafts, harvest of adequately sized conjunctival autografts may result in donor-site morbidity, including scarring, secondary granulation tissue, symblepharon, restricted ocular/eyelid motility, and partial or total limbal stem cell deficiency. ${ }^{12}$ For these reasons, we did not consider to disturb the remaining healthy conjunctiva to reduce the defect size with additional manipulations, such as conjunctival flap or partial primary closure. Moreover, the patients may refuse to have their healthy eye operated on for donor purposes. On the other hand, thick mucosal grafts, such as buccal or labial tissue, are associated with an unsatisfactory cosmetic results, as they occupy large spaces in the fornix, may shrink with time, may mask regrowth of the underlying tumor, and lead to a nonconjunctival epithelial morphology. ${ }^{12}$

The high success rates of AM in ocular surface reconstruction are because of promoted epithelization, anti-inflammatory, antifibrotic, and antiangiogenic effects, and low immunogenecity. ${ }^{15}$ The promotion of epithelization is implemented by the basement membrane of AM that serves as a substrate for normal migration, differentiation, and adhesion of epithelial cells, and prevents epithelial apoptosis. ${ }^{16}$ Moreover, the epithelium of AM stimulates healing of the ocular surface by producing several growth factors, such as epidermal growth factor, keratinocyte growth factor, hepatocyte growth factor, and basic fibroblast growth factor. ${ }^{17}$ The anti-inflammatory activity of AM might be explained by the presence of receptor antagonist of inflammation mediators including interleukin-1 and regulation of the production of activin. ${ }^{18}$ The suppression of the transforming growth factor $\beta$ signaling and the inhibition of myofibroblastic differentiation of normal fibroblasts are responsible for the antiscarring effect. ${ }^{19}$

Antiangiogenic properties of AM are thought to be determined by the expression of tissue inhibitors of metalloproteinases and endostatin, as well as by proteins that stimulate corneal epithelial growth and in this way suppress vascular endothelial cell proliferation. ${ }^{20}$ Moreover, AM has been demonstrated to elicit both goblet and non-goblet cell repopulation besides reducing inflammation. ${ }^{21,22}$

Results of AMT following excision of various conjunctival tumors, such as CIN, SCC, malignant melanoma, primary acquired melanosis, nevus, and papilloma, have been published. ${ }^{6,9-12}$ Most of the authors have reported high rates of success with few complications. However, to our knowledge, the current study presents the largest series of patients with OSSN who had cryopreserved AMT for ocular surface reconstruction with the longest-term results. Our findings are consistent with earlier reported results of several case series of AMT after conjunctival tumor excision. ${ }^{6,9-12}$ In our study, no surgical complications were observed. The postoperative period of mild discomfort and inflammation was short and effectively managed with topical steroids. Successful ocular surface reconstruction and complete epithelization of the AM were achieved in all eyes. No surface or intraocular inflammation nor persistent epithelial defect or fluorescein staining were recorded in any eyes during the follow-up period. No recurrence was observed in any eyes.

Partial limbal stem cell deficiency was found in only three eyes, despite the fact that limbal involvement was present in 18 eyes and corneal involvement was present in 14 eyes. Previous studies showed that AMT yields successful results in restoring corneal surface in patients with partial limbal stem cell deficiency. ${ }^{11,12}$ The AM probably increases expansion of stem cells by creating a noninflamed perilimbal stromal environment because of its various effects. ${ }^{12}$

As a conclusion, AMT permits more generous margins with a healthy ocular surface in extensive conjunctival OSSN. It is an effective method of reconstruction following OSSN excision, cryotherapy of surgical wound margins, corneal epitheliectomy with absolute alcohol application, and lamellar sclerectomy. In most cases, complete healing of the ocular surface can be achieved without any clinically significant complications and with good cosmetic and functional results.

\section{Summary}

\section{What was known before}

- Ocular surface reconstruction with amniotic membrane in ocular surface neoplasias is a choice of treatment.

\section{What this study adds}

- Ocular surface reconstruction with amniotic membrane in ocular surface neoplasias is a very effective choice of treatment with minor complications. Moreover, this technique allows excision with more generous margins to decrease recurrence rate.

\section{Conflict of interest}

The authors declare no conflict of interest. 


\section{References}

1 Shields JA, Shields CL. Premalignant and malignant lesions of the conjunctival epithelium. In: Shields JA, Shields CL (eds). Eyelid, Conjunctival, and Orbital Tumor: An Atlas and Textbook. LippincottWilliams \&Wilkins Co: Philadelphia, PA, USA, 2008, pp 286-305.

2 Kiire CA, Srinivasan S, Karp CL. Ocular surface squamous neoplasia. Int Ophthalmol Clin 2010; 50: 35-46.

3 Shields CL, Demirci H, Karatza E, Shields JA. Clinical survey of 1643 melanocytic and nonmelanocytic conjunctival tumors. Ophthalmology 2004; 111: 1747-1754.

4 Shields CL, Shields JA. Tumors of the conjunctiva and cornea. Surv Ophthalmol 2004; 49: 3-24.

5 Napora C, Cohen EJ, Genvert GI, Presson AC, Arentsen JJ, Eagle RC et al. Factors associated with conjunctival intraepithelial neoplasia: a case control study. Ophthalmic Surg 1990; 21: 27-30.

6 Asoklis RS, Damijonaityte A, Butkiene L, Makselis A, Petroska D, Pajaujis M et al. Ocular surface reconstruction using amniotic membrane following excision of conjunctival and limbal tumors. Eur J Ophthalmol 2011; 21: 552-558.

7 De Rotth A. Plastic repair of conjunctival defects with fetal membrane. Arch Ophthalmol 1940; 23: 522-525.

8 Prabhasawat P, Barton K, Burkett G, Tseng SC. Comparison of conjunctival autografts, amniotic membrane grafts, and primary closure for pterygium excision. Ophthalmology 1997; 104: 974-985.

9 Tseng SCG, Prabhasawat P, Lee SH. Amniotic membrane transplantation for conjunctival surface reconstruction. Am J Ophthalmol 1997; 124: 765-774.

10 Dua SH, Azuara-Blanco A. Amniotic membrane transplantation. Br J Ophthalmol 1999; 83: 748-752.

11 Espana EM, Prabhasawat P, Grueterich M, Solomon A, Tseng SCG. Amniotic membrane transplantation for reconstruction after excision of large ocular surface neoplasias. Br J Ophthalmol 2002; 86: 640-645.

12 Gündüz K, Ucakhan OO, Kanpolat A, Gunalp I. Nonpreserved human amniotic membrane transplantation for conjunctival reconstruction of extensive ocular surface neoplasias. Eye 2006; 20: 351-357.

13 Azuaro-Blanco A, Pillai CT, Dua HS. Amniotic membrane transplantation for ocular surface reconstruction. Br J Ophthalmol 1999; 83: 399-402.

14 Shields JA, Shields CL, DePotter P. Surgical management of conjunctival tumors: the 1994 Lynn B. McMahan Lecture. Arch Ophthalmol 1997; 115: 808-815.

15 Dua HS, Gomes JA, Kinng AJ, Maharajan VS. The amniotic membrane in ophthalmology. Surv Ophthalmol 2004; 49: 51-77.

16 Grueterich M, Tseng SCG. Human limbal progenitor cells expanded on intact amniotic membrane ex-vivo. Arch Ophthalmol 2002; 120: 783-790.

17 Koizumi NJ, Inatomi TJ, Sotozono CJ, Fullwood NJ, Quantock AJ, Kinoshita S. Growth factor mRNA and protein in preserved human amniotic membrane. Curr Eye Res 2000; 20: 173-177.

18 Lee SB, Li DQ, Tan DTH, Meller DC, Tseng SCG. Suppression of TGF $\beta$ signalling in both normal conjunctival fibroblasts and pterygial body fibroblasts by amniotic membrane. Curr Eye Res 2000; 20: 325-334.

19 Tseng SCG, Dq Li, Max X. Suppression of transforming growth factor isoforms, TNF- $\beta$ receptor II, and myofibroblast differentiation in cultured human corneal and limbal fibroblasts by amniotic membrane matrix. J Cell Physiol 1999; 179: 325-335.

20 Hao Y, Ma DH, Hwang DG, Kim WS, Zhang F. Identification of antiangiogenic and antiinflammatory proteins in human amniotic membrane. Cornea 2000; 19: 348-352.

21 Barabino S, Rolando M. Amniotic membrane transplantation elicits goblet cell repopulation after conjunctival reconstruction in a case of severe ocular cicatricial pemphigoid. Acta Ophthalmol Scand 2003; 81: 68-71.

22 Meller D, Dabul V, Tseng SC. Expansion of conjunctival epithelial progenitor cells on amniotic membrane. Exp Eye Res 2002; 74: 537-545. 